\title{
Prevalence of mutations in thiopurine S-methyltransferase gene among Slovak IBD patients
}

\author{
Desatova $\mathrm{B}^{2}$, Hlavaty $\mathrm{T}^{1}$, Balakova $\mathrm{D}^{3}$, Pav I ${ }^{2}$, Celec $\mathrm{P}^{3,4}$, Gregus $\mathrm{M}^{6}$, Zakuciova $\mathrm{M}^{7}$, Hlista $\mathrm{M}^{8}$, \\ Horakova $\mathrm{M}^{9}$, Kadasi $\mathrm{L}^{3,5}$, Huorka $\mathrm{M}^{1}$, Batovsky $\mathrm{M}^{2}$
}

Department of Gastroenterology, University Hospital Bratislava Petrzalka, Slovakia. barboradesat@yahoo.com

\begin{abstract}
Background: Thiopurine S-methyltransferase (TPMT) plays an important role in the metabolism of thiopurines. It has been suggested that TPMT genetic polymorphisms lead to dose-related hematopoetic toxicity. Since there are major ethnic differences in the prevalence of particular TPMT variants, it is important for each country to study their own prevalence in order to estimate the role of TPMT variants-related thiopurines toxicity in the particular inflammatory bowel disease (IBD) population.

Aims: The aim of this study was to determine the frequency of the four most common allelic variants of TPMT gene in the population of Slovak IBD patients.

Methods: TPMT genetic polymorphisms (TPMT*2, TPMT*3A, TPMT*3B, TPMT*3C) were amplified using PCR and consequently genotyped on genetic analyzer. The allele frequencies of particular allelic variants were calculated and compared with other Caucasian populations reported so far.

Results: Three hundred and thirty IBD patients were included; 196/132/2 Crohn's disease/ulcerative colitis/ unclassified colitis, $180(55 \%)$ males. Ninety-three percent of patients were homozygous for wild type TPMT variant. Heterozygous genotype of any of the studied polymorphisms was present in $6 \%$ of patients, only one patient was homozygous for TPMT*3A allele $(0.3 \%)$. The most prevalent mutant allele was TPMT*3A (3.2 \%). The distribution of the most common allelic variants of TPMT gene among Slovak IBD patients were in accordance with previously reported prevalence in Caucasian populations.

Conclusion: This study shows the prevalence of TPMT genetic polymorphisms in the Slovak IBD patient's population. As in other Caucasian populations, the most common mutant allelic variant is TPMT*3A, and the prevalence of homozygosity is relatively low (Tab. 3, Ref. 16). Full Text in PDF www.elis.sk.

Key words: thiopurine S-methyltransferase, thiopurines toxicity, genetic polymorphisms.
\end{abstract}

Thiopurines such as azathioprine (AZA) and 6-mercaptopurine (6-MP) are immunosuppressive drugs effective in inducing and maintaining the remission of inflammatory bowel disease (IBD) (14). Thiopurine S-methyltransferase (TPMT) is a cytosolic enzyme that plays an important role in the metabolism of thiopurines. It catalyses the S-methylation of thiopurine drugs $(3,12,16)$. The final products of metabolism of thiopurines are 6-thioguanine nucleotides (6-TGN) responsible for clini\begin{tabular}{l}
\hline${ }^{1}$ Department of Internal Medicine V, Division of Gastroenterology and \\
Hepatology, University Hospital Bratislava Ruzinov, Slovakia, ${ }^{2}$ Depart- \\
ment of Gastroenterology, University Hospital Bratislava Petrzalka, Slo- \\
vakia, ${ }^{3}$ Department of Molecular Biology, Comenius University, Bratisla- \\
va, Slovakia, ${ }^{4}$ Institute of Molecular Biomedicine, Comenius University, \\
Bratislava, Slovakia, ${ }^{5}$ Institute of Molecular Physiology and Genetics, \\
Slovak Academy of Sciences, Bratislava,Slovakia, ${ }^{6} \mathrm{KM}$ Gastroenterology \\
centre Nitra, Slovakia, ${ }^{7}$ Department of Internal Medicine I, Division of \\
Gastroenterology and Hepatology, University Hospital Kosice, Slovakia, \\
${ }^{8}$ Department of Internal Medicine, Hospital Trencin, Slovakia, ${ }^{9}$ Depart- \\
ment of Internal Medicine II, Division of Gastroenterology and Hepato- \\
logy, University Hospital Martin, Slovakia \\
Address for correspondence: B. Desatova MD, PhD, Dept of Gastroentero-
\end{tabular} logy, University Hospital Bratislava, Antolska 11, SK-851 07 Bratislava, Slovakia.

Phone: +421.911540280 cal benefit as well as for side effects of thiopurines (4). TPMT enzyme activity is under the control of genetic polymorphisms that have been extensively studied in the past (15). It has been suggested that TPMT genetic polymorphisms are associated with reduced TPMT enzyme activity that can lead to dose-related hematopoetic toxicity in patients treated with thiopurines. TPMT enzyme is encoded by an approximately $34 \mathrm{~kb}$ gene located on chromosome 6 (6p22.3) and contains 10 exons and 9 introns $(2,16)$. To date, at least 24 mutant alleles are known that have been reported in association with reduced TPMT enzyme activity (2). The most common mutant alleles such as TPMT*3A, TPMT*2, TPMT*3C and TPMT*3B are detected in $80-95 \%$ of Caucasian population (16). The molecular defect in TPMT*3A allele is caused by 2 nucleotide transition mutations $(\mathrm{G} 460 \rightarrow \mathrm{A}$ and $\mathrm{A} 719 \rightarrow \mathrm{G})$; the defect in TPMT*2 contains the mutation $\mathrm{G} 238 \rightarrow \mathrm{C}$, while TPMT*3C and TPMT*3B alleles carry $\mathrm{A} 719 \rightarrow \mathrm{G}$ and $\mathrm{G} 460 \rightarrow \mathrm{A}$ mutations, respectively. Ethnic differences in distribution of TPMT mutant alleles have been described $(9,11,16)$. Since there are major ethnic differences in the prevalence of particular TPMT variants, it is important for each country to study their own prevalence in order to estimate the role of TPMT variants-related thiopurines toxicity in the particular IBD population. 
Tab. 1. Demographic characteristic of the Slovak IBD population.

\begin{tabular}{lc}
\hline Demographic characteristic & Number of patients \\
\hline Number of patients & 330 \\
Male/Female & $180 / 150$ \\
Age (mean + range) in years & $37(17-75)$ \\
Crohn's disease/Ulcerative colitis/ Unclassified colitis & $196 / 132 / 2$ \\
\hline
\end{tabular}

Tab. 2. Prevalence of wild type homozygotes, mutant heterozygotes and mutant homozygotes among Slovak IBD patients.

\begin{tabular}{lcc}
\hline & Number of patients & $\%$ \\
\hline Number of patients & 330 & 100 \\
\hline Wild type homozygotes (TPMT $* 1 / * 1)$ & 308 & 93 \\
\hline Mutant heterozygotes & 1 & 0.3 \\
TPMT *2/*1 & 19 & 5.8 \\
TPMT *3A $/ * 1$ & 0 & 0 \\
TPMT *3B $/ * 1$ & 1 & 0.3 \\
TPMT *3C/*1 & 1 & 0.3 \\
Mutant homozygotes & 0 & 0 \\
TPMT *2/*2 & 1 & 0.3 \\
TPMT *3A $/ 3 A$ & 0 & 0 \\
TPMT *3B $/ * 3 B$ & 0 & 0 \\
TPMT *3C/*3C & 0
\end{tabular}

\section{Material and methods}

A total of 330 IBD patients treated in Slovakia between the year 2007 and 2009 were included. The only inclusion criterion was the diagnosis of IBD (Crohn's disease, ulcerative colitis or unclassified colitis) established by endoscopic, histological and radiological findings (Tab. 1-3). The mode of therapy was not taken into consideration. The most common TPMT variant alleles TPMT*2, TPMT*3A, TPMT*3B, and TPMT*3C were determinated. The allele frequencies of particular allelic variants were calculated and compared with other Caucasian populations reported so far. For descriptive statistics and frequencies, SPSS system version 15.0 was used. Genomic DNA was isolated from 1 milliliter of venous blood using the Puregene Blood Core kit (Qiagen, Hilden, Germany). Three different PCRs were used for preamplification of fragments containing polymorphisms $460 \mathrm{G}>\mathrm{A}, 719 \mathrm{~A}>\mathrm{G}$ and $238 \mathrm{G}>\mathrm{C}$, respectively. Amplifications were performed in $10 \mu \mathrm{l}$ of reaction volume containing $50 \mathrm{ng}$ of genomic DNA, 1x concentrated 5PRIME HotMasterMix (5PRIME, Hamburg, Germany) and 2.5 pmol of primers. After amplifications, PCR products were used as a template for sequencing reactions with the BigDye Terminator v 3.1 Cycle Sequencing Kit (Applied Biosystems, Foster City, USA). Electrophoresis was carried out on an ABI PRISM ${ }^{\circledR} 3100$ Genetic Analyzer (Applied Biosystems, Foster City, USA) and the obtained sequences were compared with the reference sequences using BLAST sequence alignment software.

\section{Results}

One-hundred and ninety six patients with Crohn's disease, 132 with ulcerative colitis, and 2 with unclassified colitis were included in this study. The mean age of included patients was 37 years, range $17-75$ years, 180 (55\%) patients were males, 150 (45\%) were females. Ninety three percent of patients were homo-
Tab. 3. Allelic variants of TPMT gene and their frequencies among Slovak IBD patients.

\begin{tabular}{lcc}
\hline Allelic variants & Number of alleles & $\%$ \\
\hline Number of alleles & 660 & 100 \\
TPMT *1 & 637 & 96.5 \\
TPMT *2 & 1 & 0.2 \\
TPMT *3A & 21 & 3.2 \\
TPMT *3B & 0 & 0 \\
TPMT *3C & 1 & 0.2 \\
\hline
\end{tabular}

zygous for wild type TPMT variant (TPMT*1/*1). Heterozygous genotype of any of the studied polymorphisms was present in $6 \%$ of patients, only one patient was homozygous for TPMT*3A allele $(0.3 \%)$. The most prevalent mutant allele was TPMT*3A (3.2\%). The frequency of mutant alleles TPMT*3C and TPMT*2 was $0.2 \%$. In this studied group no TPMT*3B mutant allele was detected. The distribution of the most common allelic variants of TPMT gene among Slovak IBD patients were in accordance with previously reported high prevalence of TPMT*3A variant and lower frequencies of TPMT*3C and TPMT*2 in Caucasian populations.

\section{Discussion}

TMPT genotyping is the way of how to determine the group of IBD patients treated with thiopurines at high risk of serious side effects such as bone marrow toxicity (13). Patients heterozygous or homozygous for mutant TPMT allele (low TPMT activity) are at higher risk of developing severe hematopoetic toxicity while treated with standard dose of thiopurines (10). In our cohort, $93 \%$ of patients were homozygous for wild type TPMT, $6 \%$ had a heterozygous genotype of any of the studied polymorphisms, and only one patient had a homozygous genotype for mutant variant TPMT*3A. There are big ethnic differences and also ethnic heterogeneity in the distribution of TPMT mutant variants. It is important for each country to determine their own prevalence by reason of estimating the role of TMPT mutant alleles-related thiopurines toxicity in each particular IBD population. This is the TPMT genotype study that shows the distribution of TPMT mutant variants among Slovak IBD patients. The allele frequencies of TPMT mutant allelic variants are comparable with those in other Caucasian and Latin-American populations. The most common allelic variant among Slovak IBD patients was TPMT*3A (3.2\%), while TPMT*2 and TPMT*3C were present only rarely $(0.2 \%)$. In Chinese, Japanese, Indian (7), Korean (9) and African populations, TMPT $* 3 \mathrm{C}$ is the most prevalent mutant allele $(5,8)$, while in Latin-American population it was not detected et al (6). On the other hand the TPMT*2 allele is present in 9.4 $\%$ of mutant alleles in British population, while in Slovak IBD patients it is very rare, and was not present in any of Ghanaian subjects (1). The TMPT*3B was not detected in Slovak IBD population at all. The mutant allele TPMT*7 has been recently found in European Caucasian population, TPMT*8 in African Americans, and TPMT*6 in Korean population (8). They seem to be rare in Caucasian population but they have not been investigated in our cohort. 


\section{2-284}

In conclusion, we have determined that the distribution of mutant TPMT variants in Slovak IBD patients are in accordance with previously reported distribution in other Caucasian populations.

\section{References}

1. Ameyaw MM, Collie-Duguid ESR, Powrie RH, Ofori-Adjei D, McLeod HL. Thiopurine methyltransferase alleles in British and Ghanaian populations. Hum Mol Genet 1999; 8 (2): 367-370.

2. Cao Q, Zhu Q, Shang Y, Gao M, Si J. Thiopurine Methyltransferase Gene Polymorphisms in Chinese Patients with Inflammatory Bowel Disease. Digestion 2009; 79 (1): 58-63.

3. Egan LJ, Derijks LJJ, Hommes DW. Pharmacogenomics in inflammatory bowel disease. Clin Gastroenterol Hepatol 2006; 4 (1): 21-28.

4. Gurwitz D, Rodríguez-Antona C, Payne K et al. Improving pharmacovigilance in Europe: TPMT genotyping and phenotyping in the UK and Spain. Eur J Hum Genet 2009; 17 (8): 991-998.

\section{Hon YY, Fessing MY, Pui ChH, Relling MV, Krynetski EY, Evans} WE. Polymophism of the thiopurines S-methyltransferase gene in AfricanAmericans. Hum Mol Genet 1999;8 (2): 371-376.

6. Isaza C, Henao J, López AM, Cacabelos R. Allelic variants of the thiopurine methyltransferase (TPMT) gene in the Colombian population. Methods Find Exp Clin Pharmaco 2003; 25 (6): 423-429.

7. Kham SKY, Soh ChK, Liu TCh et al. Thiopurine S-methyltransferase activity in three major Asian populations: a population based study in Singapore. Eur J Clin Pharmacol 2008; 64 (4): 373-379.

8. Kubota T, Chiba K. Frequencies of thiopurne S-methyltransferase mutant alleles (TPMT*2, *3A, *3B and *3C) in 151 healthy Japanese subjects and the inheritance of TPMT*3C in the family of a propositus. Br J Clin Pharmacol 2001; 51 (5): 475-477.

9. Lennard L. TPMT in the treatment of Crohn's disease with azathioprine. Gut 2002; 51 (2): 143-146.

10. Leung M, Piatkov I, Rochester C, Boyages SC, Leong RWL. Normal thiopurine methyltransferase phenotype testing in a Crohn disease patient with azathioprine induced myelosuppression. Intern Med J 2009; 39 (2): 121-126.

11. Otterness DM, Szumlanski CL, Wood TC, Weinshilboum RM. Human Thiopurine Methyltransferase Pharmacogenetics. Kindred with a Terminal Exon Splices Junction Mutation That Results in Loss of Activity. J Clin Invest 1998; 101 (5): 1036-1044.

12. Takatsu N, Matsui T, Murakami Y et al. Adverse reactions to azathioprine cannot be predicted by thiopurine S-methyltransferase genotype in Japanese patients with inflammatory bowel disease. J Gastroenterol Hepatol 2009; 24 (7): 1258-1264.

13. Teml A, Schaeffeler E, Schwab M. Pretreatment determination of TPMT - state of the art in clinical practice. Eur J Clin Pharmacol 2009; 65 (3): 219-221.

14. Winter JW, Gaffney D, Shapiro D et al. Assessment of thiopurine methyltransferase enzyme activity is superior to genotype in predicting myelosuppression following azathioprine therapy in patients with inflammatory bowel disease. Aliment Pharmacol Ther 2007; 25 (9): 1069-1077.

15. Yates ChR, Krynetski EY, Loennechen T. Molecular diagnosis of thiopurine S-methyltrasnferase deficiency: genetic basis for azathioprine and mercaptopurine intolerance. Ann Intern Med 1997; 126 (8): 608-614.

16. Zhou S. Clinical pharmacogenomics of Thiopurine S-methyltransferase. Curr Clin Pharmacol 2006; 1 (1): 119-128. 